\title{
Colorful Polymer Compositions with Dyed Graphene Oxide Nanosheets
}

\author{
Y. M. Shulga, ${ }^{1}$ S. A. Baskakov, ${ }^{1}$ V. E. Muradyan, ${ }^{1}$ D. N. Voylov, ${ }^{1}$ V. A. Smirnov, ${ }^{1}$ \\ A. Michtchenko, ${ }^{2}$ J. G. Cabañas-Moreno, ${ }^{2}$ K. G. Belay, ${ }^{3}$ C. A. Weatherford, ${ }^{3}$ and G. L. Gutsev ${ }^{3}$ \\ ${ }^{1}$ Institute of Problems of Chemical Physics of Russian Academy of Sciences, Chernogolovka, Moscow 142432, Russia \\ ${ }^{2}$ Instituto Politécnico Nacional, ESFM, UPALM, Avenida IPN, Edif. 9, 07738 Mexico City, DF, Mexico \\ ${ }^{3}$ Department of Physics, Florida A\&M University, Tallahassee, FL 32307, USA
}

Correspondence should be addressed to G. L. Gutsev, gennady.gutsev@famu.edu

Received 23 November 2011; Accepted 26 December 2011

Academic Editors: L. Shah and A. Tervonen

Copyright () 2012 Y. M. Shulga et al. This is an open access article distributed under the Creative Commons Attribution License, which permits unrestricted use, distribution, and reproduction in any medium, provided the original work is properly cited.

\begin{abstract}
A water suspension of graphene oxide nanosheets (GONSs) obtained via exfoliation of a graphite oxide was treated with a solution of rhodamine 6G. It was found that adding the dye results in the destruction of the GONS water suspension and the precipitation of dyed graphene oxide. The precipitate, washed out of the excess dye and subjected to a second dispersal via sonification, provides a stable suspension of dyed GONS in water or dimethylformamide. The GONS dyeing produces shifts of major absorption bands of the dye in solutions or in polymer compositions toward larger wavelengths. We also found that the stability of a dye subjected to ultraviolet irradiation increases if the dye is bound to a GONS. The increased stability resulted from excitation transfer from dye to a GO nanosheet and its subsequent reduction.
\end{abstract}

\section{Introduction}

Graphite oxide is known since the 19th century [1]. The current surge of interest to graphite oxide is due to not only its application as a prospective material for electrodes, membranes, and polymer additives [2-7] but also to a new avenue which opened after the discovery of the unique properties of graphene [8-10]. Contrary to many other carbon materials, graphite oxide is hydrophilic and is capable of forming colloidal solutions in water, spirits, and other polar solvents, where GO splits into thin sheets of up to one carbon layer thickness [11-13]. Graphite oxide "solubility" is due to a large number of oxygen-containing groups chemically bound to graphene layers. The presence of these groups deteriorates the mechanical and conducting properties of GO nanosheets with respect to those of graphene. A hydrazine processing restores [14-16] the GONS $\pi$-system and results in properties similar to those of graphene. Generally, the GO nanosheets possess [17] sites with different reaction abilities; therefore, it is difficult to predict beforehand the results of interactions between a GONS and different molecules.
This letter describes the products of interactions between a GONS and the dye rhodamine 6G (R6G, $\left.\mathrm{C}_{28} \mathrm{H}_{31} \mathrm{ClN}_{2} \mathrm{O}_{3}\right)$ and then discusses some properties of this product. The goal of this work was to produce dyed GONSs in transparent polymer compositions. We prepared films of polymethylmethacrylate (PMMA) modified using both dye and dyed GONSs. The film samples were characterized using spectral methods.

\section{Experimental Details}

Our GO samples were prepared according to Hummers and Offeman's method [18] using a procedure whose details are described elsewhere [19]. Suspensions were prepared by mixing GO $(100 \mathrm{mg})$ with water $(100 \mathrm{~mL})$ in a glass vessel followed by ultrasound irradiation for $30 \mathrm{~min}$ with an applied power of $150 \mathrm{~W}$. The suspension was centrifuged for $20 \mathrm{~min}$ at $2700 \mathrm{~g}$ in order for large particles to be removed. The precipitate mass did not exceed several mg. The suspension obtained possessed a homogeneous brown color and showed no change for a quite long time. The water 


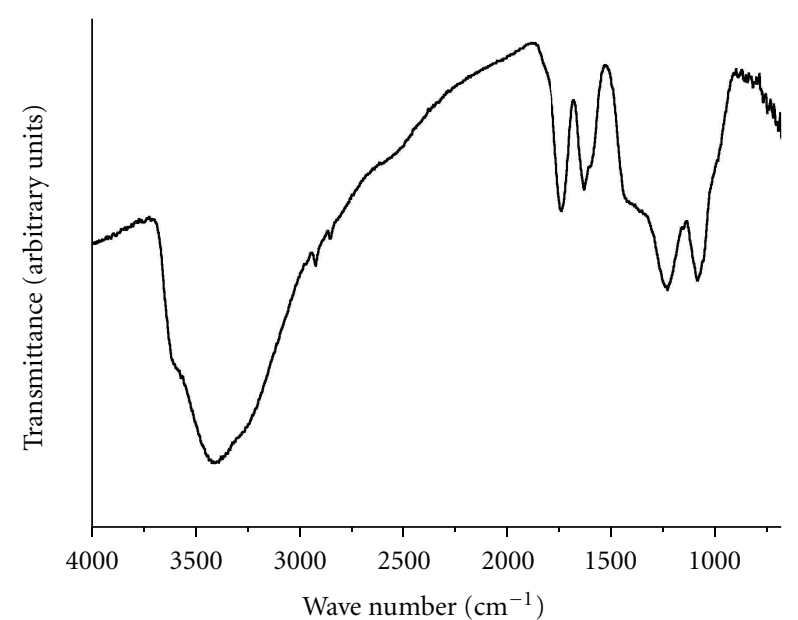

FIGURE 1: The IR spectrum of the film formed after drying of the GO water suspension droplet deposited over the crystal of UTAR accessory.

suspension of GO was mixed with a dye solution at room temperature. The precipitate, which formed, was filtered and washed several times with water in order to remove excess dye. Then dyed GONSs were subjected to the second dispersal via sonification in water or dimethylformamide (DMF). In this way, stable transparent suspensions were obtained whose color was different from the colors of both initial GO suspension and the dye solution.

In order to prepare a film with the dyed GONS additives, we chose PMMA, which is the most transparent among the known polymers and is soluble in DMF. The dye and dyed GONSs, as a solution (first), a suspension (second) in DMF, or as a mixture of DMF and water, were added to the solution of PMMA in DMF. Films of pure PMMA and PMMA with additives were obtained in air at the temperature of $70^{\circ} \mathrm{C}$ by drying the corresponding solutions in Petri's plates with flat bottoms. The samples were irradiated using a full-light high-pressure mercury lamp (DRSh-1000) for $30 \mathrm{~min}$. The samples were mounted $50 \mathrm{~cm}$ away from the lamp in order to avoid having the sample absorb excessive heat.

IR spectra in the range $675-4000 \mathrm{~cm}^{-1}$ were registered using a Fourier spectrometer-a Perkin Elmer Spectrum 100 equipped with UATR. The GO sheet's thickness was estimated using the scanning tunnel microscopy (STM) using a NTEGRA Aura, NT-MDT microscope. The UV-viz spectra in the range $190-1100 \mathrm{~nm}$ were measured using a spectrophotometer (Perkin Elmer Lambda 45). For visualization, we used a digital camera Canon Power Shot 5G.

\section{Results and Discussion}

Figure 1 presents the IR spectrum of a film formed after drying a suspension droplet deposited over the crystal of UATR accessory of the IR spectrometer. This spectrum is similar to the spectra obtained in two previous studies $[19,20]$ and possesses a substantially low intensity for the absorption band at $930-980 \mathrm{~cm}^{-1}$ with respect to the spectra obtained

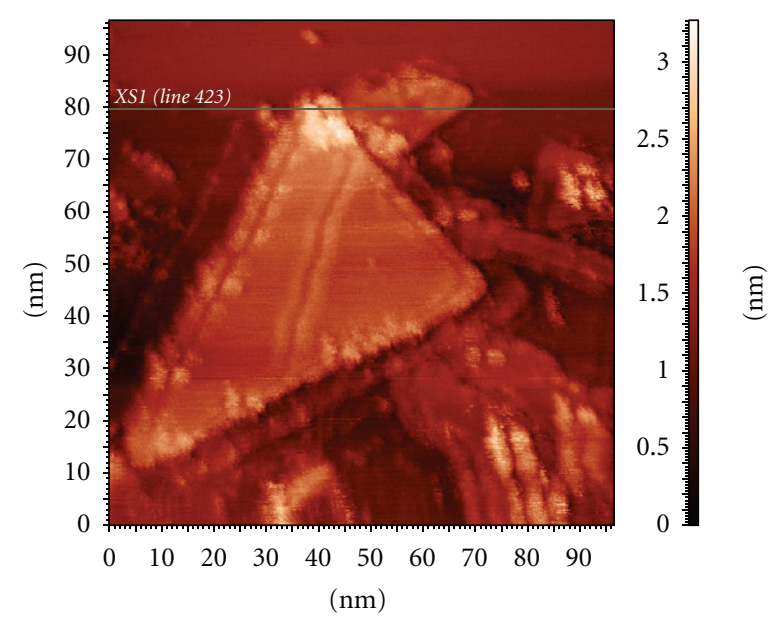

(a)

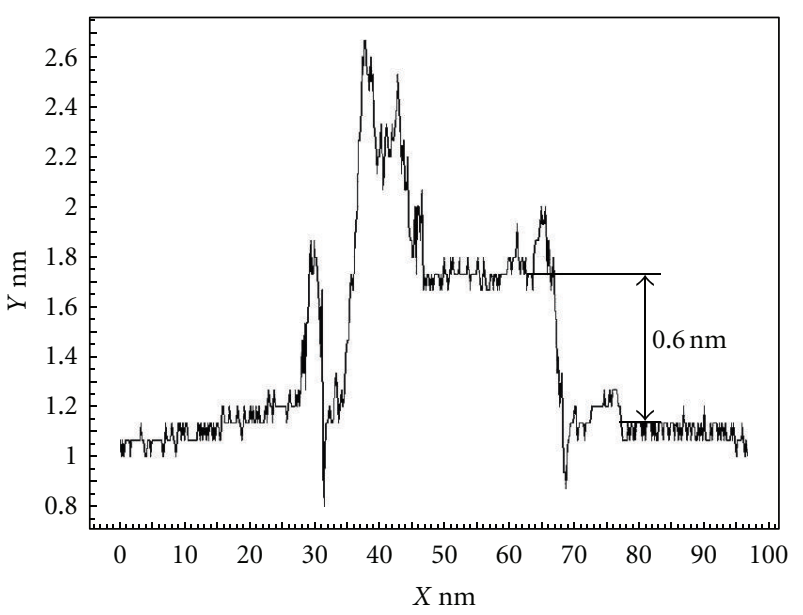

(b)

FIGURE 2: The STM image of the film formed after a droplet of GO suspension in water deposited on the surface of highly oriented pyrolytic graphite has dried.

in two previous studies $[21,22]$. This band was related to vibrations of epoxide groups. A detailed description of the band assignment in the IR spectra of films obtained by precipitation of GONS suspensions can be found elsewhere [23]. Particles in the centrifuged water suspension are flat sheets with the width of about $0.6 \mathrm{~nm}$ according to the STM data presented in Figure 2. This result matches the results obtained previously $[14,20,24]$. Therefore, the measured properties of the samples obtained allow one to consider them as containing GO nanosheets.

The successive steps of the GONS dyeing are reflected in Figure 3. As is seen, the water suspensions of the initial and dyed GONS are transparent and the color of the dyed suspension is different from the color of the dye solution in water. Note that the destruction of a GO suspension resulting from the added dye solution occurs within a few minutes. Thus, one has a simple way to produce the GONS precipitation from water suspensions by applying a proper dye. The use of centrifuges for this purpose requires very high 


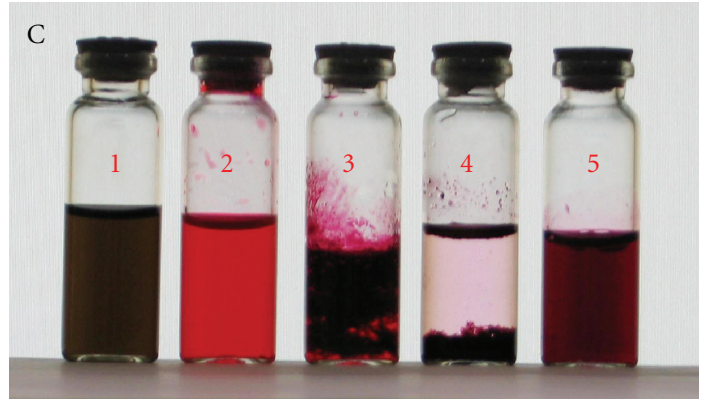

Figure 3: Photo images of glass ampoules filled in (1) the water suspension of initial GO nanosheets, (2) the water solution of rhodamine $6 \mathrm{G},(3)$ the product of interactions of the contents of the first and second ampoules immediately after the reaction, (4) the reaction precipitate product from ampoule 3 washed with water, and (5) the water suspension of dyed GO nanosheets.

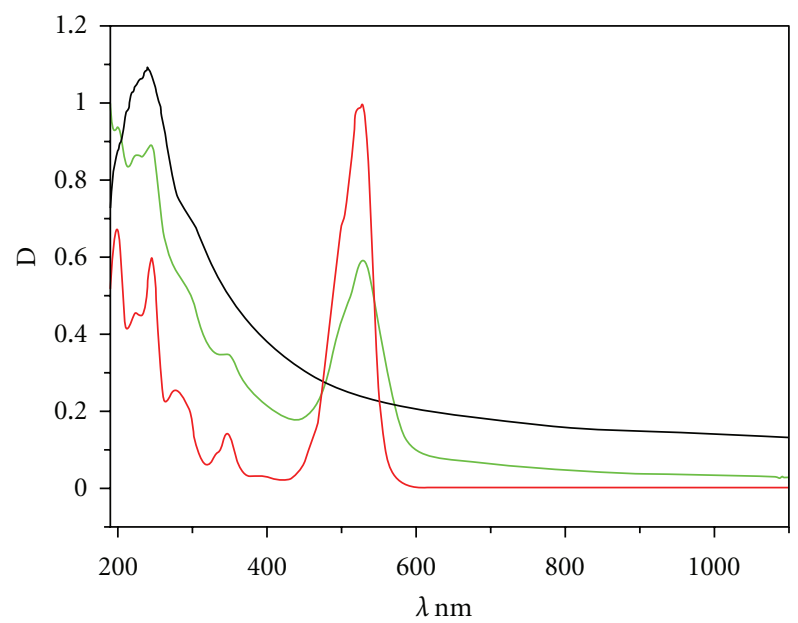

Figure 4: Optical spectra of the GO water suspension (black color), water solution of rhodamine 6G (green color), and water suspension of dyed GO nanosheets.

accelerations. According to our observations, $5700 \mathrm{~g}$ is not sufficient for the full GONS precipitation.

The shape of our optical spectrum of GO water suspension displayed in Figure 4 is similar to that obtained previously [25-27]. The band with the maximum at 228$230 \mathrm{~nm}$ is associated with an excitation of $\pi$-plasmons [25, $28,29]$ in carbon structures, where the number of $\pi$ electrons per atom is substantially smaller than 1 . Minor displacements of this band obtained in the studies above appear to be related to different concentrations of $\pi$-electrons participating in plasma oscillations. The GONS dyeing results in a shift of $4 \mathrm{~nm}$ of the dye main band to longer wavelengths.

Many IR absorption bands related to R6G are widened or displaced in the spectra of the dyed GONS presented in Figure 5. The band at $847 \mathrm{~cm}^{-1}$, marked with the star in the figure, practically vanished. Adding R6G to a transparent PMMA film results in a homogeneous coloring of the film (see Figure 6). The coloring of PMMA films with the GONS

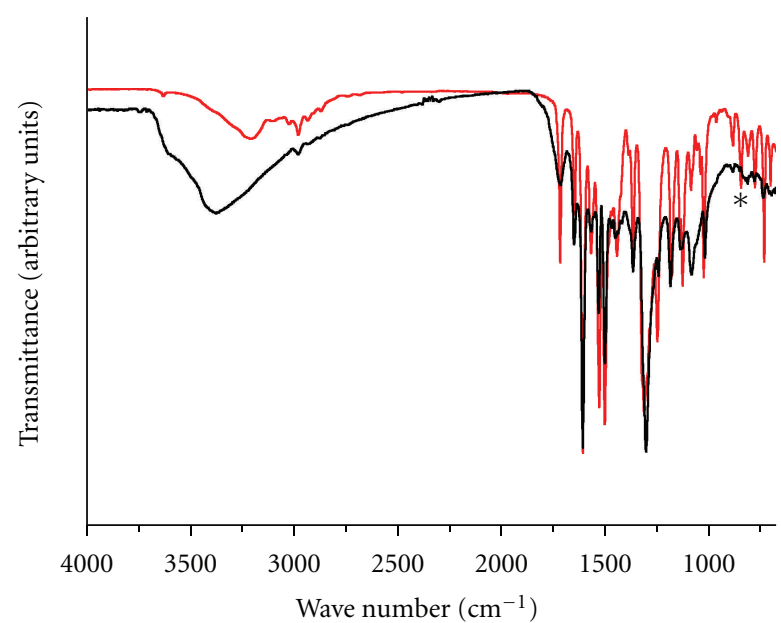

FIGURE 5: IR spectra of rhodamine 6G (red color) and GO nanosheets dyed with rhodamine 6G.

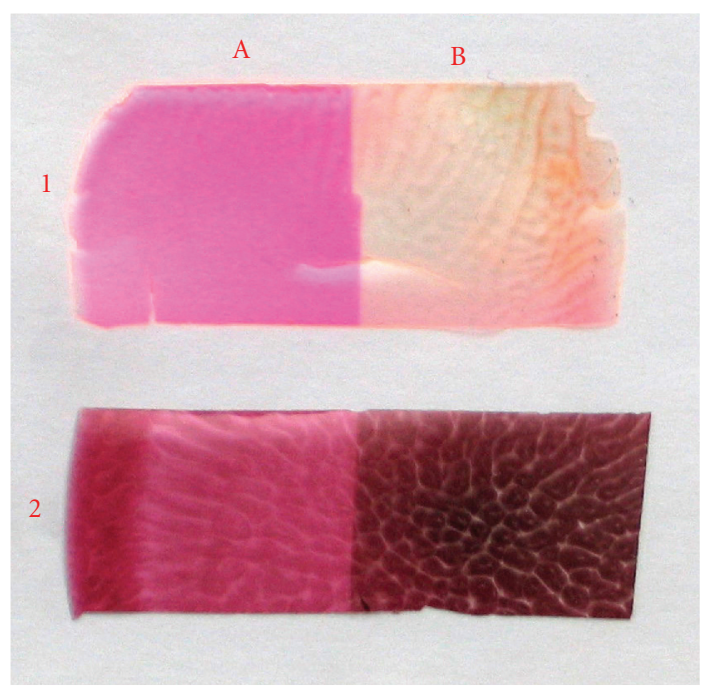

Figure 6: Photo images of the PMMA films: (1) corresponds to the film dyed with rhodamine 6G; (2) corresponds to the film dyed with colored GONS, before (A) and after (B) irradiation.

additives (both dyed and undyed) is visually inhomogeneous. Microscopic observations showed that this inhomogeneity is not related to a segregation of GO particles. Its appearance might be related to the high rate of the film drying.

An ultraviolet irradiation of PMMA films dyed with R6G results in a fast discoloration of the films as shown in Figure 6. It is not true for the PMMA films with dyed GONS additives. Under the same irradiation conditions, the latter films became darker but the color does not disappear. This difference can be explained on the basis of the spectra in the visible light range. As is seen from Figure 7, the darkening of the PMMA films with dyed GONSs additives corresponds to the optical density surge in the whole wavelength range except for the wavelengths related to the absorption, which defines the color of the dye. Such a background increase in 


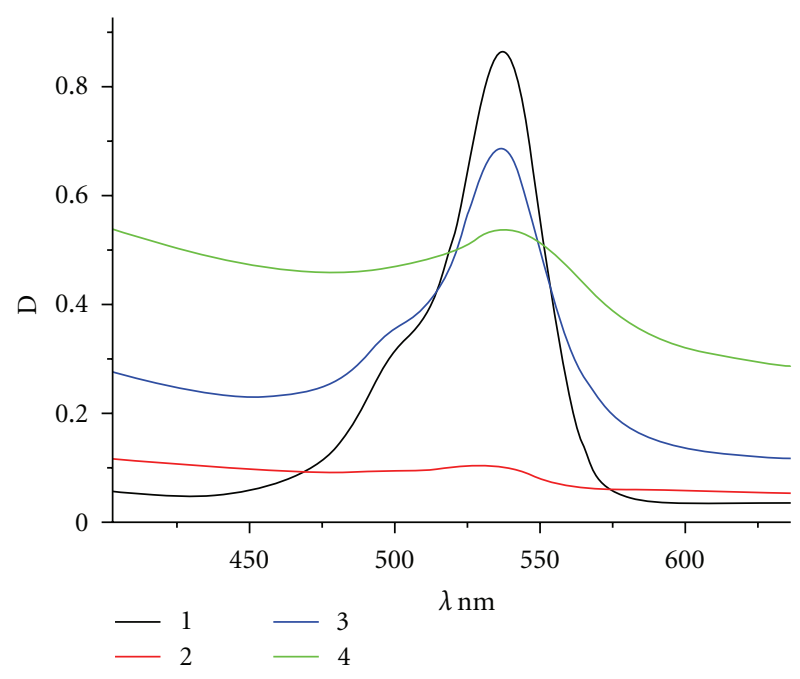

Figure 7: Optical spectra of the PMMA films: (1) corresponds to the initial film dyed with rhodamine 6G; (2) corresponds to the film in 1 after irradiation; (3) corresponds to the initial film with dyed GONS additives; (4) corresponds to the film in 3 after irradiation.

the optical density can be related to the GO nanoparticle reduction $[30,31]$. The integral intensity of the main peak of the R6G, in the case of the PMMA films with dyed GONS, decreased 2.7 times due to the irradiation. Irradiation under the same conditions of the films dyed with clean R6G lead to a diminishing intensity of this peak by 27 times! One concludes that the dye stability increases 10 times after the dye is bound to a GONS. The main reason of this increased stability is the excitation transfer from the dye to a GO nanosheet with subsequent reduction.

In conclusion, we note that similar experiments were performed with other dyes such as Neutral Red, Brilliant Green, Fluorexon, Murexide, and Bengal Pink A. From this group, only Neutral Red and Brilliant Green behaved as rhodamine $6 \mathrm{G}$, that is, their addition to the GONS water suspension led to a precipitation. The other dyes just colored the mixture without any precipitation.

\section{References}

[1] B. C. Brodie, "Sur le poids atomique du graphite," Annales de Chimie et de Physique, vol. 59, pp. 466-472, 1860.

[2] R. Yazami and P. Touzain, "Lithium—graphite oxide cells Part III: effect of origin and oxidation of graphite on batteries performances," Synthetic Metals, vol. 12, no. 1-2, pp. 499-503, 1985.

[3] M. Mermoux, R. Yazami, and P. Touzain, "Lithium-graphitic oxide cells part IV: influence of electrolyte and cathode composition," Journal of Power Sources, vol. 20, no. 1-2, pp. 105110, 1987.

[4] T. Cassagneau and J. H. Fendler, "High density rechargeable lithium-ion batteries self-assembled from graphite oxide nanoplatelets and polyelectrolytes," Advanced Materials, vol. 10, no. 11, pp. 877-881, 1998.

[5] T. Hwa, E. Kokufuta, and T. Tanaka, "Conformation of graphite oxide membranes in solution," Physical Review A, vol. 44, no. 4, pp. R2235-R2238, 1991.
[6] X. Wen, C. W. Garland, T. Hwa et al., "Crumpled and collapsed conformations in graphite oxide membranes," Nature, vol. 355, no. 6359, pp. 426-428, 1992.

[7] F. F. Abraham and M. Goulian, "Diffraction from polymerized membranes: flat vs. crumpled," Europhysics Letters, vol. 19, pp. 293-296, 1992.

[8] K. S. Novoselov, A. K. Geim, S. V. Morozov et al., "Electric field in atomically thin carbon films," Science, vol. 306, no. 5696, pp. 666-669, 2004.

[9] K. S. Novoselov, A. K. Geim, S. V. Morozov et al., "Twodimensional gas of massless Dirac fermions in graphene," Nature, vol. 438, no. 7065, pp. 197-200, 2005.

[10] A. K. Geim and K. S. Novoselov, "The rise of graphene," Nature Materials, vol. 6, no. 3, pp. 183-191, 2007.

[11] M. Hirata, T. Gotou, S. Horiuchi, M. Fujiwara, and M. Ohba, "Thin-film particles of graphite oxide 1: high-yield synthesis and flexibility of the particles," Carbon, vol. 42, no. 14, pp. 2929-2937, 2004.

[12] M. Hirata, T. Gotou, and M. Ohba, "Thin-film particles of graphite oxide. 2: preliminary studies for internal micro fabrication of single particle and carbonaceous electronic circuits," Carbon, vol. 43, no. 3, pp. 503-510, 2005.

[13] T. Szabó, A. Szeri, and I. Dékány, "Composite graphitic nanolayers prepared by self-assembly between finely dispersed graphite oxide and a cationic polymer," Carbon, vol. 43, no. 1, pp. 87-94, 2005.

[14] S. Stankovich, D. A. Dikin, R. D. Piner et al., "Synthesis of graphene-based nanosheets via chemical reduction of exfoliated graphite oxide," Carbon, vol. 45, no. 7, pp. 1558-1565, 2007.

[15] J. R. Lomeda, C. D. Doyle, D. V. Kosynkin, W. F. Hwang, and J. M. Tour, "Diazonium functionalization of surfactantwrapped chemically converted graphene sheets," Journal of the American Chemical Society, vol. 130, no. 48, pp. 16201-16206, 2008.

[16] V. C. Tung, M. J. Allen, Y. Yang, and R. B. Kaner, "Highthroughput solution processing of large-scale graphene," Nature Nanotechnology, vol. 4, no. 1, pp. 25-29, 2009.

[17] R. Y. N. Gengler, K. Spyrou, and P. Rudolf, "A roadmap to high quality chemically prepared Graphene," Journal of Physics D, vol. 43, no. 37, Article ID 374015, 19 pages, 2010.

[18] W. S. Hummers and R. E. Offeman, "Preparation of graphitic oxide," Journal of the American Chemical Society, vol. 80, no. 6, p. 1339, 1958.

[19] V. E. Muradyan, M. G. Ezerskaya, V. I. Smirnova et al., "Transformation of graphite oxide at conditions of ionic hydration," Russian Journal of Organic Chemistry, vol. 12, pp. 2626-2629, 1991.

[20] Y. Si and E. T. Samulski, "Synthesis of water soluble graphene," Nano Letters, vol. 8, no. 6, pp. 1679-1682, 2008.

[21] H. K. Jeong, Y. P. Lee, M. H. Jin, E. S. Kim, J. J. Bae, and Y. H. Lee, "Thermal stability of graphite oxide," Chemical Physics Letters, vol. 470, no. 4-6, pp. 255-258, 2009.

[22] L. J. Cote, R. Cruz-Silva, and J. Huang, "Flash reduction and patterning of graphite oxide and its polymer composite," Journal of the American Chemical Society, vol. 131, no. 31, pp. 11027-11032, 2009.

[23] Y. M. Shulga, V. M. Martynenko, V. E. Muradyan, S. A. Baskakov, V. A. Smirnov, and G. L. Gutsev, "Gaseous products of thermo- and photo-reduction of graphite oxide," Chemical Physics Letters, vol. 498, no. 4-6, pp. 287-291, 2010.

[24] H. C. Schniepp, J. L. Li, M. J. McAllister et al., "Functionalized single graphene sheets derived from splitting graphite oxide," 
Journal of Physical Chemistry B, vol. 110, no. 17, pp. 85358539, 2006.

[25] X. Sun, Z. Liu, K. Welsher et al., "Nano-graphene oxide for cellular imaging and drug delivery," Nano Research, vol. 1, pp. 203-212, 2008.

[26] M. J. Hudson, F. R. Hunter-Fujita, J. W. Peckett, and P. M. Smith, "Electrochemically prepared colloidal, oxidised graphite," Journal of Materials Chemistry, vol. 7, no. 2, pp. 301-305, 1997.

[27] S. Stankovich, R. D. Piner, X. Chen, N. Wu, S. T. Nguyen, and R. S. Ruoff, "Stable aqueous dispersions of graphitic nanoplatelets via the reduction of exfoliated graphite oxide in the presence of poly(sodium 4-styrenesulfonate)," Journal of Materials Chemistry, vol. 16, no. 2, pp. 155-158, 2006.

[28] B. W. Reed and M. Sarikaya, "Electronic properties of carbon nanotubes by transmission electron energy-loss spectroscopy," Physical Review B, vol. 64, no. 19, Article ID 195404, 13 pages, 2001.

[29] S. Attal, R. Thiruvengadathan, and O. Regev, "Determination of the concentration of single-walled carbon nanotubes in aqueous dispersions using UV-visible absorption spectroscopy," Analytical Chemistry, vol. 78, no. 23, pp. 8098-8104, 2006.

[30] D. Li, M. B. Müller, S. Gilje, R. B. Kaner, and G. G. Wallace, "Processable aqueous dispersions of graphene nanosheets," Nature Nanotechnology, vol. 3, no. 2, pp. 101-105, 2008.

[31] V. A. Smirnov, A. A. Arbuzov, Yu. M. Shul'ga et al., "Photoreduction of graphite oxide," High Energy Chemistry, vol. 45, no. 1, pp. 57-61, 2011. 

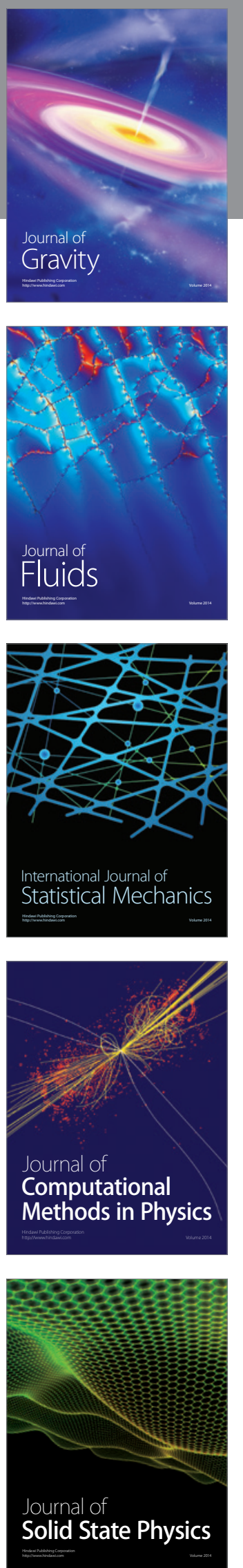
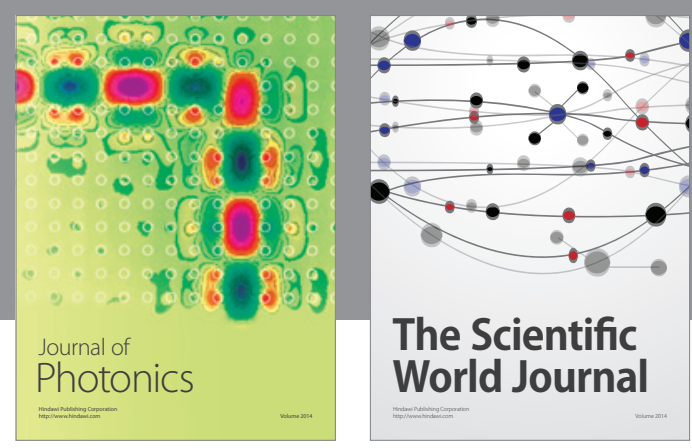

The Scientific World Journal

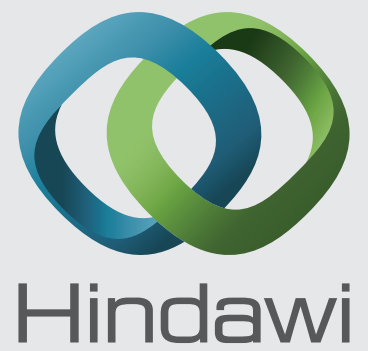

Submit your manuscripts at http://www.hindawi.com
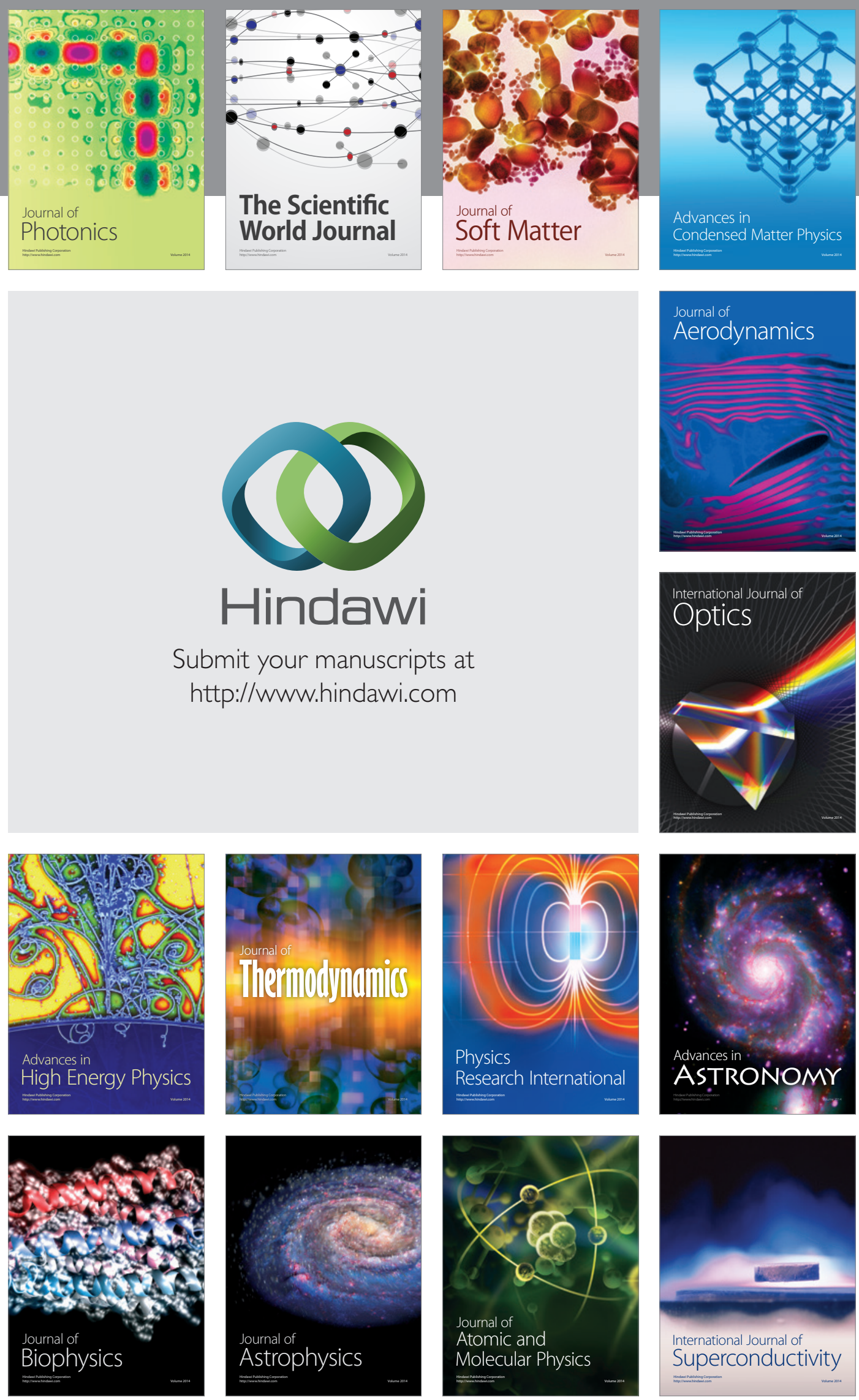
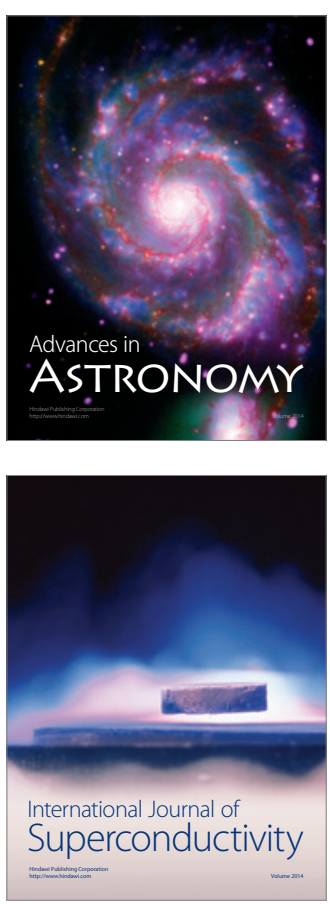